\title{
LA RESPONSABILIDAD SOCIAL DE LAS EMPRESAS
}

\section{THE SOCIAL RESPONSABILITY OF THE COMPANIES}

\author{
Alan Errol Rozas Flores* \\ Docente Asociado de la Facultad de Ciencias Contables, UNMSM
}

[Recepción: Marzo de 2009 / Conformidad: Mayo de 2009]

\section{RESUMEN}

El público quiere saber más sobre las empresas y no de manera casual sino sistemática para tomar mejores decisiones de inversión y de ahorro (balance adecuado entre la rentabilidad y los riesgos) y para evaluar la sostenibilidad de la empresa con pautas concretas sobre su legitimidad para operar: su conducta de hacer negocios y su ponderación equilibrada de temas económicos, sociales y ambientales.

Hay una íntima relación entre lo que es el desarrollo sustentable y la Responsabilidad Social de la Empresa (RSE). Las empresas declaran un compromiso con el desarrollo sustentable, porque entienden que tiene que ver con su viabilidad. Y en ello, coexisten dos grandes motivaciones: imperativos éticos y también imperativos de negocios.

En relación con lo primero, se constata la convicción que las empresas son responsables por sus impactos en la sociedad y que distintos grupos de interés (stakeholders), no sólo los inversionistas, tienen derecho a saber. En relación con lo segundo, también existe la convicción que las empresas que son más abiertas ganarán confianza de sus grupos de interés, desarrollarán una mejor reputación, tendrán más aliados y menos problemas, y así serán percibidas como administrando mejor todos sus riesgos, en otras palabras, demostrarán buen management, que ve más allá del corto plazo.

\begin{abstract}
The public wants to know more on the companies and not of casual way but systematic to take better decisions of investment and of saving (felicitous balance between the benefits and the risks) and to evaluate the sustainable of the company with rules concrete on his legitimacy to operate: his conduct to do businesses and his equilibrium balanced of economic subjects, social and environmental.

There is an intimate relation between what the development sustainable and the Social Responsibility of the Company (RSE). The companies declare a commitment with the development sustainable, because they understand that it has to see with his feasibility. And in this, coexist two big motivations; imperative ethical and also imperative of businesses.

In relation with the first, ascertains the conviction that the companies are responsible by his impact in the society and that distinct groups of interest (stakeholders), not only the investors, have right to knowledge. In relation with the second, also exists the conviction that the companies that are more open will win confidence of his groups of interest, will develop a better reputation, will have more allies and least problems, and like this will be perceived and administering better all his risks; In other words it will show good management that sees further of the short term.
\end{abstract}


Lo que mueve a la empresa es la mejor forma de hacer negocios, la que asegure esa permanencia en el tiempo. Y eso sí concilia con los imperativos éticos de muchos: el cuidado y la acción responsable del medio ambiente, la transparencia de información, el buen gobierno empresarial, la promoción de practicas de negocios sanos, respeto a los integrantes de la cadena de valor, la atención y cuidado o consideración a la gente de las empresas en aspectos sociales, de salud y de seguridad.

Palabras clave: Gestión, control interno, desarrollo sustentable, empresa, gobierno corporativo, grupos de interés, informe, negocios, responsabilidad social, sostenibilidad.
The moves to the company is the best form to do businesses, the one who ensure this permanence in the time. And this itself conciliates with the imperative ethical of many: the care and the responsible action of the means environment, the transparency of information, the good business government, the promotion of practices of healthy businesses, respect to the members of the chain of value, the attention and care or consideration to the people of the companies in social appearances, of health, of security.

Keywords: Management, Internal Control, Sustainable Development, Company, Corporative, Corporate government, Groups of interest (Stakeholders), Report, Business, Social responsibility, Sustainable. 


\section{INTRODUCCIÓN}

La responsabilidad social es una filosofía, una actitud o forma de ver la vida que implica que tomemos en cuenta el efecto que nuestras acciones y decisiones tienen sobre el entorno físico y social. En otras palabras, ser socialmente responsable significa ser consciente del daño que nuestros actos pueden ocasionar a cualquier individuo o grupo social.

Por tanto, la responsabilidad social empresarial no es más que la extensión de la responsabilidad individual que tiene todo ciudadano hacia su entorno físico y social, esto es lo que se ha venido a llamar ciudadanía corporativa. El concepto de ciudadanía corporativa implica que la empresa, al igual que el ciudadano, tiene deberes y derechos para con la sociedad y los debe cumplir y respetar.

\section{LA RESPONSABILIDAD SOCIAL DE LAS EMPRESAS (RSE)}

Hasta el momento no hay una sola definición de responsabilidad social de las empresas (RSE) que haya sido mundialmente aceptada.

Existen varias definiciones propuestas sobre RSE y que han tenido distintos grados de aceptación en la comunidad académica y empresarial. A continuación se presentan las más reconocidas:

- "La responsabilidad social empresarial es el compromiso que asume una empresa para contribuir al desarrollo económico sostenible por medio de colaboración con sus empleados, sus familias, la comunidad local y la sociedad en pleno, con el objeto de mejorar la calidad de vida”.

(World Business Council for Sustainable Development - WBCSD, Suiza).

- "La responsabilidad social empresarial es el conjunto de prácticas empresariales abiertas y transparentes basadas en valores éticos y en el respeto hacia los empleados, las comunidades y el ambiente". (Prince of Wales Business Leadership Forum (PWBLF), Inglaterra).

- "La responsabilidad social empresarial se define como la administración de un negocio de forma que cumpla o sobrepase las expectativas éticas, legales, comerciales y públicas que tiene la sociedad frente a una empresa".

(Business for Social Responsibility (BSR), Estados Unidos).

- La responsabilidad social empresarial es hacer negocios basados en principios éticos $y$ apegados a la ley. La empresa (no el empresario) tiene un rol ante la sociedad, ante el entorno en el cual opera. La decisión de hacer estos negocios rentables, de forma ética y basados en la legalidad es realmente estratégico, ya que con esto se generará:

- Mayor productividad: a través de mejores condiciones para el cliente interno que conduce a mejor retención de talentos y por ende menores índices de rotación;

- Lealtad del cliente: satisfaciendo sus necesidades, empezando por proveerle un lugar donde pueda transmitir sus necesidades y quejas. Además de calidad y precio, los clientes empiezan a demandar información de las condiciones de producción, las certificaciones que tiene el producto, entre otras; $y$

- Acceso a mercados: por cumplimiento de estándares y certificaciones exigidas por actores externos, incluyendo consumidores.

- Credibilidad: la empresa que es respetuosa de las personas, comunidades, medio ambiente y la sociedad en 
su conjunto proyecta una reputación que le garantiza mayor sostenibilidad en el tiempo, reduciendo riesgos, anticipándose a situaciones que pueden afectar la empresa, mayor agilidad para reaccionar $y$ adaptarse $y$ generando confianza. (Instituto CENTRARSE de Guatemala).

En este sentido, se puede concluir que la responsabilidad Social Corporativa (RSC), también llamada Responsabilidad Social Empresarial (RSE), es la contribución activa y voluntaria de las empresas al mejoramiento social, económico y ambiental. Bajo este concepto de administración y de management se engloban un conjunto de prácticas, estrategias y sistemas de gestión empresariales que persiguen un nuevo equilibrio entre las dimensiones económica, social y ambiental. La importancia que en la actualidad los diferentes participantes de la sociedad y negocios otorgan a la RSC ha determinado que ésta sea una actividad estratégica en la competencia comercial. La responsabilidad social corporativa es el origen de las diferentes acciones que se realizan para alcanzar un fin, la sostenibilidad.

La responsabilidad social corporativa es el conjunto de principios, políticas, programas y acciones de respeto al entorno social y natural dirigidos a incorporar en la estrategia empresarial los valores y expectativas de los diversos públicos internos y externos con el fin de incrementar el valor de marca y la reputación de la empresa.

\section{ORIGEN DE LA RSE}

El concepto de responsabilidad social corporativa o responsabilidad social de la empresa surge en Estados Unidos a finales de los años
50 y principios de los 60, a raíz de la Guerra de Vietnam y otros conflictos como el apartheid.

Despierta el interés en los ciudadanos que comienzan a creer que, a través de su trabajo en determinadas empresas o comprando algunos productos, están colaborando con el mantenimiento de determinados regímenes políticos, o con ciertas prácticas políticas o económicas éticamente censurables.

En consecuencia, la sociedad comienza a pedir cambios en los negocios y una mayor implicación del entorno empresarial en los problemas sociales.

La responsabilidad social de la empresa (RSE), también denominada responsabilidad social corporativa (RSC), es un término que hace referencia al conjunto de obligaciones y compromisos, legales y éticos, tanto nacionales como internacionales, que se derivan de los impactos que la actividad de las organizaciones producen en el ámbito social, laboral, medioambiental y de los derechos humanos. De igual forma que hace medio siglo las empresas desarrollaban su actividad sin tener en cuenta el marketing o que hace tres décadas la calidad no formaba parte de las orientaciones principales de la actuación empresarial, hoy en día las empresas son cada vez más conscientes de la necesidad de incorporar las preocupaciones sociales, laborales, medioambientales $\mathrm{y}$ de derechos humanos, como parte de su estrategia de negocio.

\section{LA TEORÍA DE LOS GRUPOS DE INTERÉS (STAKEHOLDERS)}

Los grupos de interés o stakeholders están constituidos por grupos específicos de personas - accionistas, trabajadores, consumidores, comunidad, etc.-, a los que afectan, 
de una u otra manera, las acciones y decisiones de la empresa. Es decir, los stakeholders son aquellos que tienen algún interés legal, ecológico, económico, cultural, etc. en las operaciones y decisiones de la empresa.

La teoría de los grupos de interés (stakeholders) considera que existen distintos grupos de personas que pondrían verse afectadas de manera distinta de acuerdo a sus propios intereses por las acciones y decisiones de las empresas. Esto implica la existencia de exigencias sociales, que surgen en cada grupo de interés y que la empresa debe atender con acciones de responsabilidad social. Mediante la identificación de los grupos de interés, la empresa puede poner "nombres y caras" a los miembros de la sociedad que son prioritarios para ella y ante quienes debe responder.

Cuando se habla de la dirección o del manejo de los grupos de interés, nos referimos al proceso que emprenden los directivos de las empresas para evitar que sus objetivos choquen con los objetivos y expectativas de los distintos grupos de interés. Pero, ¿cómo evitar un conflicto de intereses? El reto está en la dirección de los stakeholders, que deberán encontrar la manera de que los principales grupos de interés de la empresa logren sus objetivos mientras los demás grupos también estén satisfechos.

Existen cinco principales grupos de interés reconocidos como prioritarios por la mayoría de las empresas, independientemente de la industria a la que pertenezcan y de su tamaño o ubicación. Estos son:

- Propietarios.

- Empleados.

- Clientes (incluye proveedores).

- Comunidades locales (incluye al gobierno).

- Sociedad en general (incluye al medio ambiente).

\section{LA PIRÁMIDE DE LA RSE}

El concepto de responsabilidad social de la empresa (RSE) puede variar con el tiempo y ser sustituido por otros enfoques diferentes, tales como correspondencia social, actuación social, política pública, ética o dirección de grupos de interés. El de toda la sociedad es el de definir las clases de responsabilidades que los directivos y las empresas tienen hacia los grupos con los que realizan transacciones e interactúan más frecuentemente.

La teoría de la pirámide de la RSE desarrollada por Carroll (1991) plantea cuatro clases de responsabilidades sociales de las empresas, vistas como cuatro noveles de una pirámide. Hay responsabilidades que se encuentran en el fondo de la pirámide y que son, por tanto, la base sobre la que se apoya otro tipo de responsabilidades. Las cuatro clases de responsabilidades son: económicas, legales, éticas y filantrópicas.

1. Responsabilidades económicas Constituyen la base de la pirámide y son entendidas como la producción de bienes y servicios que los consumidores necesitan y desean. Como compensación por la entrega de estos bienes y servicios, la empresa debe obtener una ganancia aceptable en el proceso.

2. Responsabilidades legales

Tienen que ver con el cumplimiento de la ley y de las regulaciones estatales, así como con las reglas básicas según las cuales deben operar los negocios.

3. Responsabilidades éticas

Se refieren a la obligación de hacer lo correcto, justo y razonable, así como de evitar o minimizar el daño a los grupos de interés (empleados, consumidores, medio ambiente y otros). Estas responsabilidades implican respetar aquellas 
actividades y prácticas que la sociedad espera, así como evitar las que sus miembros rechazan, aun cuando éstas no se encuentren prohibidas por la ley.

4. Responsabilidades filantrópicas Comprenden aquellas acciones corporativas que responden a las expectativas sociales sobre la buena ciudadanía corporativa. Estas acciones incluyen el involucramiento activo de las empresas en actividades o programas que promuevan el bienestar social y mejoren la calidad de vida de la población. La diferencia entre las responsabilidades éticas y las filantrópicas está en que las primeras surgen porque la empresa quiere cumplir con las normas éticas de la sociedad; mientras que las segundas no son una norma esperada en un sentido ético o moral, sino que representan más bien una actividad voluntaria de parte de las empresas, aun cuando siempre existe la expectativa social de que éstas las sigan.

En resumen, de acuerdo a la teoría de la pirámide, la RSE implica el cumplimiento simultáneo de las responsabilidades económicas, legales, éticas y filantrópicas. En otras palabras, la RSE debe llevar a la empresa a obtener ganancias, obedecer la ley, ser ética y comportarse como un buen ciudadano corporativo.

\section{LA RESPONSABILIDAD SOCIAL DE LAS EMPRESAS DESDE EL ENTORNO SOCIAL}

Bajo el concepto de la RSE se esconde una amalgama de iniciativas que tratan de introducir la dimensión ética y social en la empresa, ya que, más allá de la empresa misma, gobiernos y organizaciones de índole muy diversa han desempeñado y lo siguen haciendo un papel muy importante en el planteamiento de iniciativas e instrumentos útiles para promocionar la RSE.

Tanto la Comisión Europea como el gobierno federal de Estados Unidos han planteado líneas de actuación para promover la RSE. A escala europea la promoción de la RSE se ha establecido sobre las bases de la voluntariedad promulgadas en la comunicación de la Comisión de julio de 2001, conocida como Libro Verde de la CE para el Fomento de la RSE. La Ley Sarbanes-Oaxley de 2002, dictada en Estados Unidos, parte de un enfoque diferente para exigir de las empresas, por ley, unos requisitos mínimos de transparencia informativa relativos a la dimensión ética de la compañía con el fin de prevenir escándalos derivados de una posible gestión fraudulenta.

En los mercados financieros también se han creado espacios restringidos de inversión donde cotizan las empresas que asumen criterios de publicidad en sus actuaciones para la sostenibilidad y el buen gobierno. Así, el FTSE4GOOD y el Dow Jones Sustainability Index incorporan los criterios de la RSE en el mercado de acciones más importante del planeta. Otra alternativa diferente, aunque también dentro del mismo sector, es la inversión financiera realizada a partir de criterios éticos. Con altibajos que son propios de la evolución de las cotizaciones bursátiles, globalmente sigue incrementándose el número de partícipes en los fondos de inversión elaborados a partir de criterios de responsabilidad social y medioambiental.

$\mathrm{El}$ accionista puede decidir que restringe su inversión en función de sus preferencias negativas alrededor de áreas de negocio como las bebidas alcohólicas o positivas a favor de una empresa que preste atención a los derechos humanos o que combata el 
uso de mano de obra infantil en su cadena productiva. Incluso vemos, en los países de la Unión Europea, que cada vez es mayor la oferta financiera que proporcionan los llamados bancos éticos, que restringen el uso de los depósitos realizados por sus clientes a finanzas o proyectos social y ecológicamente sostenibles.

Por otro lado, siguiendo la pauta establecida por las normativas ISO de gestión de la calidad y sostenibilidad medioambiental, en los últimos años ha proliferado el número de certificaciones que cubren las relaciones internas de la empresa con su personal, la atención y el respeto hacia los derechos humanos en los países donde opera la empresa o, directamente, las pautas de buen gobierno con las que ésta se gestiona. En el mismo sentido, un gran número de organizaciones privadas ha promovido instrumentos de medición de la RSE con indicadores concretos cuyo objetivo es ser prueba de fe del avance surgido en la implementación de los criterios de responsabilidad social. Actualmente, el modelo de Global Reporting Initiative, con sus indicadores de RSE, es el que está sirviendo como base para la elaboración de las memorias sociales y medioambientales de las grandes empresas.

Además, existen otras iniciativas muy diferentes que pretenden garantizar al gran público que los productos que adquieren incorporan en su elaboración y distribución criterios de responsabilidad social y ecológica. El uso de las etiquetas de comercio responsable o de producto ecológico se dirige a los consumidores que están dispuestos a pagar un sobreprecio a cambio de saber que la cadena de suministro de la empresa productora ha sido retribuida justamente o que los productos que van a ingerir se han elaborado sin pesticidas ni edulcorantes artificiales. Es muy interesante comprobar el creciente porcentaje de clientes que actúan al margen de las dos variables tradicionales: calidad y precio, y hacen valer sus valores sociales y medioambientales en el momento de adquirir un producto. El caso de Gran Bretaña, dentro de la Unión Europea, es particularmente paradigmático en este aspecto.

\section{LA RESPONSABILIDAD SOCIAL DE LAS EMPRESAS DESDE UNA PERSPECTIVA CONTABLE}

En el mundo actual, el concepto de imagen fiel como principal criterio para definir y construir la información económico financiera de las empresas para los terceros ajenos a ésta se muestra insuficiente $o$, al menos, incapaz de aproximar la realidad de la empresa a aquellos que esperan obtener, con su análisis, un buen diagnóstico de presente y futuro. Los diferentes criterios contables utilizados en el registro de las transacciones económicas (los criterios de devengo, de prudencia, de continuidad de negocio, etcétera, en los que se basan las cuentas anuales de las sociedades, junto con el informe de auditoría) han seguido el camino de esta doctrina, si bien en los últimos tiempos, forzados por la globalización y los escándalos relacionados con la fiabilidad de esta información, las entidades reguladoras de los mercados financieros han determinado unos requisitos de información adicionales que complementan a los ya existentes. Por tanto, nos situamos en un escenario en el cual el mundo económico solicita información sobre aspectos que hasta fechas relativamente recientes no se consideraban relevantes.

Definido el concepto de responsabilidad social, cabría realizar la puntualización de que ser socialmente responsable no sólo significa cumplir con las obligaciones jurídicas, 
sino también ir más allá de su cumplimiento, es decir, invertir más en capital humano, en el entorno y en las relaciones con los interlocutores. Ante este nuevo escenario, los posicionamientos contrarios y favorables se han explicitado y formalizado planteándose, en consecuencia, argumentos diversos. Considerando que en cualquier caso existe una responsabilidad social de la empresa, encontramos las siguientes posiciones:

Posiciones positivas. Son las que entienden que la maximización del beneficio debe alcanzarse atendiendoúnicas y exclusivamente a obligaciones legales. Como representante de estas ideas destacaremos al liberal M. Friedman. La responsabilidad se limitaría a un cumplimiento de la legalidad (normativa) y en caso de que ésta exista, queda reducida a un acto de naturaleza voluntaria.

Asociados a posiciones positivas encontramos argumentos matizados, que consideran que la responsabilidad se reduce al cumplimiento de unos requisitos económicos y legales, siempre que el marco legal sea suficiente. La culpa de que los empresarios aparezcan como socialmente irresponsables sólo la tiene el gobierno, que no garantiza la existencia de un verdadero Estado de derecho, es decir, reglas de juego clara y aplicable por igual a todos.

Posiciones negativas. Según éstas, la empresa es responsable de sus actuaciones en la medida en que éstas pueden llegar a causar algún efecto en el entorno en el que opera. Aquí encontramos a Blomstrom. En este caso, pues, existe una responsabilidad de tipo moral.

La obligación de la empresa, según la teoría neoclásica, establece que ésta tiene como único objetivo la "maximización instantánea de beneficios"; hoy esta teoría está supera$\mathrm{da}$, y se entiende que la empresa tiene como objetivo proporcionar bienes y servicios a la sociedad respetando en todo momento "los derechos reconocidos por la sociedad en la que vive y los valores que esta sociedad comparte", y para alcanzar este fin es necesario el beneficio económico, que no es el fin sino el medio de la actividad empresarial (Camita, 1989).

Por lo tanto, si bien es cierto que el objetivo de la empresa es la maximización del beneficio, su búsqueda deberá tener en consideración (y en ningún caso deberá ignorar) a los diferentes grupos de interés que se relacionan y/o dependen de ella, en la medida en que son ellos los que legitiman en última instancia su supervivencia en la sociedad.

Con frecuencia el ordenamiento jurídico y su cumplimiento efectivo (sobre todo en países en vías de desarrollo) no consiguen su objetivo de garantizar la práctica de unos valores éticos «legales». Esto explicaría que hoy se constate, en el mundo el trabajo infantil, el deterioro del medio ambiente, el olvido de la salud de las comunidades más pobres, la falta de una justa remuneración y la inexistencia de derechos del consumidor. En realidad, aquí surgen otros tipos de «valoresprácticas $\gg:$ la defensa de los grupos de intereses económicos, la necesidad de atraer la inversión extranjera a cambio de políticas de dumping medioambiental y laboral, etcétera.

Por tanto, visto desde una u otra perspectiva, y conscientes de la realidad en la que nos movemos (podemos decir, por tanto, que la existencia de la responsabilidad social es un hecho cierto), es necesario definir los parámetros que nos permitirán establecer cuál es el nivel de compromiso que una empresa asume en las distintas vertientes en las que interactúa con su entorno. Parece claro, como ya hemos indicado, que la información financiera tradicional es insuficiente; así, por ejemplo, y sin entrar en términos muy complejos, la definición de activos intangibles no 
permite reflejar aquellos aspectos propios de la empresa que son una fuente generadora de valor, soslayando por tanto unos elementos que configurarían un balance invisible. No obstante, el único campo en el que los contables han intentado evaluar de forma sistemática los recursos que no figuran en el balance tal vez sea el de los recursos humanos.
En la medida en que los recursos humanos $\mathrm{u}$ otros activos intangibles no queden incluidos en el balance de la empresa, la responsabilidad sobre éstos y otros parecidos se reparte por toda la empresa, al no existir un responsable o «controlador». Como ejemplo de elementos intangibles presentamos el siguiente cuadro:

\begin{tabular}{|l|l|l|}
\hline & \multicolumn{1}{|c|}{ RECURSOS TANGIBLES } & \multicolumn{1}{c|}{ RECURSOS INTANGIBLES } \\
\hline $\begin{array}{l}\text { Recursos que figuran en el } \\
\text { balance }\end{array}$ & Instalaciones & Fondo de comercio \\
& Equipos & Concesiones \\
& Existencias & Arrendamientos financieros \\
\hline & Personal & Ética y compromiso social \\
Recursos que no figuran en el & Plan estratégico \\
balance & Cecnología & Imagen de la empresa \\
& Consejo de administración & Relaciones con los accionistas \\
& Sistemas información para la dirección & Relaciones con el mundo financiero \\
& & Relaciones con clientes e instituciones \\
& & Cultura de la empresa \\
\hline
\end{tabular}

\section{RECOPILACIÓN, GESTIÓNY FIABILIDAD DE INFORMACIÓN}

Actualmente, las grandes corporaciones multinacionales son las pioneras en el mundo de la responsabilidad social corporativa. No obstante, existen unos elementos que es preciso considerar de forma crítica en los informes o documentos de responsabilidad social, de sostenibilidad, etcétera, como son los datos (ratios, índices) que se nos ofrecen.

La elaboración de indicadores sociales internacionales no se justifica únicamente por la necesidad de las empresas de disponer de nuevos enfoques en lo que se refiere a cuadros de mando para la dirección, sino para poder ofrecer datos al exterior sobre sus prácticas sociales.

Antes de concretar indicadores, es necesario mencionar la dificultad de las grandes corporaciones para efectuar la recogida de datos; esta dificultad se concreta en dar respuesta a una doble exigencia: ofrecer datos globales y que obedezcan a realidades loca- les. Esta situación supone consolidar datos con orígenes, referencias legales e institucionales diferentes, con el consiguiente riesgo de interpretaciones y resultados erróneos, así como disponer de unos sistemas de información que den respuesta a la demanda de información.

En este sentido el Informe de Desarrollo Sostenible 2003 de Vivendi Universal establece que "apoyándose en el tronco común de la 01T, el grupo deja a todas las entidades operacionales una gran autonomía en materia de gestión de recursos humanos".

También el Informe de Desarrollo Sostenible 2003 de Veolia Environnement puede, gracias a su dispositivo de recogida mundial de datos sociables, disponer de las principales evoluciones y las grandes tendencias en referencia a sus 309,563 asalariados que están empleados en unas 2.000 sociedades. Este dispositivo, mediante un filtro de más de 500 colaboradores repartidos por el mundo, permite obtener anualmente más de 100 indicadores sociales. 
Otro ejemplo de la complejidad para obtener datos está constituido por el criterio que recoge Danone en su Informe de Responsabilidad Social y Medio Ambiente del 2002: "Las comparaciones internacionales en materia de seguridad en el trabajo han de realizarse con mucha prudencia. Según los países, la forma de cálculo varía y algunos accidentes considerados como - benignos - no se contabilizan, sobre la base de razones de prácticas culturales diferentes. Así, la frecuencia de los accidentes de trabajo es más elevada en Europa que para el conjunto del grupo (excepto Asia)".

Debe tenerse en cuenta que para interpretar mejor los datos está el hecho de incorporar antecedentes del sector emitidos por organismos independientes y reconocidos que permitan conocer el posicionamiento de las empresas con relación a determinados aspectos. Por ejemplo, es recomendable incorporar en las memorias de las empresas eléctricas el volumen de emisión de gases emitido y clasificarlo en un ranking, como también la cualidad de sus emisiones. Evidentemente, las empresas no aceptarían esta situación, a pesar de que para los demandantes de información podrían ser muy significativos.

\section{LA RESPONSABILIDAD SOCIAL DE LAS EMPRESAS EN PAÍSES EN DESARROLLO}

La RSE cubre un amplio rango de temas relacionados con la conducta, desde la gobernabilidad corporativa y la protección ambiental, hasta temas de inclusión social, derechos humanos y desarrollo económico nacional. En el caso del sector de inversión privado en países de bajos y medianos ingresos, el énfasis colocado en cada uno de estos temas puede variar, y algunas veces difieren de las prioridades de inversionistas y empresas en mercados más desarrollados.

En países de bajos y medianos ingresos, el interés está creciendo sobre la posible alineación de las bondades de las actividades de la RSE con las prioridades del sector público. Por ejemplo, los gobiernos de los países en desarrollo están comenzando a ver a la RSE como un medio de promover las estrategias de desarrollo sustentable, como un componente de sus competitividades nacionales para competir por la inversión extranjera directa y para posicionar sus exportaciones globalmente, y para mejorar el enfoque de metas hacia la lucha contra la pobreza.

Actualmente, muchos países están presenciando cambios dramáticos en sus instituciones públicas y privadas. Mientras que las fuerzas de los mercados están tomando control del sector educativo, en el lugar de trabajo de la información económica, el aprendizaje de por vida es más importante. Como consecuencia, los elementos de la educación primaria, secundaria y terciaria son considerados, y las empresas están tomando un mayor interés en las actividades de las instituciones educativas. Esto da lugar a preguntas sobre la naturaleza de la relación entre el sector corporativo y educativo.

\section{CÓMO EJERCEN LAS ORGANIZACIONES SU RESPONSABILIDAD SOCIAL}

Cuando satisfacen las expectativas que tienen los diferentes grupos de interés sobre su comportamiento, contribuyendo a un desarrollo social y ambientalmente sostenible sin dejar de lado el desarrollo económicamente viable.

Disminuir riesgos administrativos de la empresa, aumentar la reputación, posicio- 
narse como empresa líder del ramo, aumentar la transparencia del reporte corporativo, identificar los grupos de interés, otorgar mayor confianza en la validez de la información no financiera a los inversionistas, son algunos de los puntos que se pueden mejorar al establecer o fortalecer la responsabilidad social corporativa.

\section{COMPARACIÓN ENTRE GOBIERNO CORPORATIVQYY RESPONSABILIDAD SOCIAL CORPORATIVA}

Los nuevos contenidos de gestión surgen del diálogo o de los compromisos acordados entre la empresa y los grupos de presión (lobbies o stakeholders). Para la organización la dimensión de estos colectivos ha ido creciendo a lo largo del tiempo. En sus inicios el único tercero al que se servía eran los accionistas. Así, por ejemplo, todos los Códigos del Buen Gobierno voluntarios aplicados a este fin, elaborados con anterioridad a 2001 ponían como cliente más importante al propio accionista y los refuerzos realizados posteriormente se encaminan hacia un incremento de la transparencia. Los Códigos de Ética van encaminados hacia un colectivo mucho más amplio ya que incluye a clientes.

Sin embargo, la presión ejercida por la comunidad se ha incrementado significativamente a lo largo de las últimas dos décadas. Para ilustrar esta transformación cabe mencionar que en el Código de Ética de Enron se consideraba como terceros a tener en cuenta a los clientes y a los proveedores.

A finales de la pasada década el concepto se ha sofisticado dividiéndose en dos grupos, los internos y los externos, a los grupos de interés; así se aprecia en la Estructura de Gobierno Corporativo, según King's Report.
Este Código de Buena Conducta para las empresas que cotizan en la Bolsa Sudafricana resulta revolucionario ya que incluye a terceros no considerados en otras Normativas de Gobierno Corporativo más recientes como la ley Sarbanes Oxley.

Sorprendentemente se acerca desde el punto de vista del Gobierno Corporativo al grupo de terceros que hoy se consideran clave en el proceso de Responsabilidad Social Empresarial porque incluye a colectivos o individups que pueden influir en la actividad de una organización o que son afectados por la misma. Sin embargo, el colectivo de grupos de interés ha crecido con respecto al aportado por el Gobierno Corporativo ya que abarca a instituciones tan importantes como las organizaciones internacionales, las ONG locales y los consumidores, cuyo protagonismo en la acción social está teniendo una repercusión más relevante en la esfera mundial.

Por esta afirmación, podría parecer que la responsabilidad social se considera como la redenominación del Gobierno Corporativo, pero no es así, pues más bien supone una ampliación de este concepto. Para sustentar esto se debe abarcar la Responsabilidad Social desde varios frentes.

En primer lugar, sus componentes más importantes son:

- Establecimiento de un sistema de Gobierno Corporativo.

- Políticas de acción social.

- Políticas de sostenimiento del medio ambiente de acuerdo a la naturaleza de la empresa.

- Transparencia informativa respecto a los logros alcanzados por la empresa, sobre los que descansa hoy gran parte de la reputación corporativo.

Parece evidente que la influencia de los gobiernos se ha volcado fundamentalmente 
en el área del Gobierno Corporativo buscando una mayor transparencia en los mercados financieros y un restablecimiento de la confianza del inversor ante el auge de escándalos financieros acaecidos al principio del milenio. El aspecto normativo del mismo que la convierte a una materia de cumplimiento legal, en principio de forma voluntaria para llegar a su conversión en normativa cuando los escándalos financieros como la ley Sarbanes Oxley, que impuso la publicación del Informe anual de Gobierno Corporativo a las empresas que cotizan en la Bolsa.

Por un lado, la noción de Gobierno Corporativo encaja bien dentro de los asuntos habituales de gestión manejados por la Alta Dirección pero puede abarcar fuertemente los asuntos tratados por la Responsabilidad Social Corporativa (Véase la Triple Cuenta de Resultados del Informe King II de Sudáfrica). Por otro lado, los conceptos de Responsabilidad Social Corporativa no han avanzado tan lejos como el Gobierno Corporativo en acordar un conjunto de principios.

En este entorno, el auditor interno tiene un papel reconocido oficialmente. Recordemos que el informe King les reconoce un rol esencial en el Gobierno Corporativo y por ende en la responsabilidad social corporativa. De aqui surge el rol integrador de la auditoría interna en el sistema de gestión de riesgos y en cuyo seno se debe integrar esta nueva materia para que sea aceptada y adoptada dentro de la cultura de la empresa.

\begin{tabular}{|l|}
\hline $\begin{array}{c}\text { ILUSTRACIÓN } 2 \text { "TRIPLE CUENTA DE RESULTADOS” } \\
\text { DEL INFORME KING II DE SUDÁFRICA }\end{array}$ \\
\hline Resultado económico financiero \\
\hline La excelencia de la calidad de bienes y productos \\
\hline La cultura corporativa \\
\hline El medio ambiente \\
\hline La prevención de riesgos laborales \\
\hline La ética y la responsabilidad social \\
\hline La globalización o la internacionalización de la compañía \\
\hline La inversión en I + D \\
\hline
\end{tabular}

\section{EVALUACIÓN Y MEDICIÓN DE LA RESPONSABILIDAD SOCIAL DE LAS EMPRESAS}

Cuando una empresa entra en contacto con el tema de la responsabilidad social, una de las interrogantes principales que surgen es la relacionada con la medición de la misma. También lo ha sido durante el desarrollo e investigación del tema desde los ámbitos empresarial y académico. Inicialmente, esta interrogante intentó ser absuelta por las organizaciones de manera individual, de modo que cada una de las empresas interesadas generó algún tipo de mecanismo de medición o manifestación de la labor social de la empresa. Con el paso de los años, sin embargo, se ha establecido criterios nacionales e internacionales para determinar cuán socialmente responsable es una empresa, de manera que se pueda realizar comparaciones reales y claras.

En general, la responsabilidad social de las empresas (RSE) debe evaluarse sobre la base de principios. Los principios más reconocidos y aceptados hasta ahora, son los siguientes:

\section{Los 10 Principios del Global Compact de la Organización de las Naciones Unidas}

1. Apoyar y respetar la protección de los derechos fundamentales.

2. Asegurarse de no ser cómplices en la vulneración de los derechos humanos.

3. Apoyar la libertad de afiliación y el reconocimiento efectivo del derecho a la negociación colectiva.

4. Apoyar la eliminación de toda forma de trabajo forzoso o realizado bajo coacción.

5. Apoyar la erradicación del trabajo infantil.

6- Apoyar la abolición de las prácticas de discriminación en el empleo y la ocupación. 
7. Mantener un enfoque preventivo que favorezca el medio ambiente.

8. Fomentar el desarrollo y difusión de tecnologías respetuosas con el medio ambiente.

9. Fomentar las iniciativas que promuevan una mayor responsabilidad ambiental; favorecer el desarrollo y la difusión de las tecnologías respetuosas con la naturaleza.

10. Trabajar contra la corrupción en todas sus formas, incluidas la extorsión y el soborno.

\section{Los siete ejes de la RSE}

El Instituto Centrarse en Guatemala ha establecido siete ejes o grandes temas que enmarcan las prácticas socialmente responsables, de donde parten una serie de indicadores para establecer las mediciones. Estos son:

1. Valores y transparencia (principios éticos y valores).

2. Actores internos (condiciones y ambiente de trabajo).

3. Proveedores (márketing responsable).

4. Consumidores o clientes (márketing responsable).

5. Medio ambiente (protección del medio ambiente).

6. Comunidad (apoyo a la comunidad).

7. Estado (apoyo a la comunidad).

\section{CONCLUSIONES}

1. Con el crecimiento de la responsabilidad social empresarial, por la cual las organizaciones se comprometen a efectuar tareas para proteger el medioambiente $y$ mejorar el ámbito laboral y de la comunidad, nace la necesidad de establecer un mecanismo para evaluar estas acciones (indicadores de desempeño, económicos, ambientales y sociales dentro de los informes financieros).

2. Si bien la evaluación y medición de la responsabilidad social empresarial es interdisciplinaria, ya que participan tanto especialistas (ingenieros, químicos, sociólogos, etc.) como contadores en condición de auditores; estos últimos son los que mayor injerencia tienen.

3. El campo laboral de los contadores públicos se ha ampliado. A las tareas tradicionales, se le suman otras, como su participación en la evaluación y medición de la responsabilidad social empresarial.

4. La evaluación y medición de la responsabilidad social empresarial es una herramienta importante, tanto para mejorar la imagen de la empresa hacia afuera, como para conocerse y repensarse hacia adentro. Evaluar y medir la RSE es necesario, casi ineludible para poder competir. A medida que este concepto se vaya extendiendo, el consumidor podrá darse cuenta de qué requerimientos cumple cada empresa, comparar y elegir entre los productos y servicios, teniendo en cuenta los parámetros de responsabilidad que ofrece y asegura.

\section{REFERENCIAS BIBLIOGRÁFICAS}

1. García, C. (1999). Ética profesional de la contaduría pública, casos prácticos. México: Instituto Mexicano de Contadores Públicos, A.C.

2. Garret, T.M. Klonosky R. J. (1986). Business Ethics. 2a ed., Englewood Cliffs. Prentice-Hall.

3. Zegarra, Luis F. (1999). Causas y consecuencias económicas de la corrupción, Perú. 
4. Alcoberro, Ramón. (2007). Ética, economía y empresa: la dimensión moral de la empresa. España: Edit. Gedisa.

5. Schwalb, María M. (2006). Responsabilidad social. Perú: Centro de Investigación de la Universidad del Pacífico.

6. Ortega, Claudia. García, Emilio. Schwalb, María Matilde. (2006). Casos de responsabilidad social. Perú: Centro de Investigación de la Universidad del Pacífico.

7 .García, Emilio. Schwalb, María Matilde. (2006). Buenas prácticas peruanas de responsabilidad social empresarial, Perú: Centro de Investigación de la Universidad del Pacífico.
8. Moliner, Miguel Ángel. (1998). Marketing social: gestión de las causas sociales. $1 .^{\mathrm{a}}$ edición, España: Esic Editorial.

9. Velásquez, Manuel G. (2000). Ética en los negocios: conceptos y casos. España: Edit. Pearson Educación.

10. Documentos y Publicaciones (papers) de la Organización de las Naciones Unidas.

11. Documentos y Publicaciones (papers) de la Agencia Internacional de Desarrollo.

12. Ospina, Alexandra. (2003). "La empresa buena”. Desarrollo humano e institucional en América Latina. México, Setiembre. 